\title{
A note on "Double-diffusive Soret convection phenomenon in porous media: effect of Vadasz inertia term"
}

\author{
F. Capone ${ }^{1}(D)$ R. De Luca ${ }^{1} \cdot$ M. Vitiello ${ }^{2}$
}

Received: 30 August 2021 / Accepted: 9 September 2021

(C) The Author(s) 2021

\begin{abstract}
In this short paper, some analytical results found in "Double-diffusive Soret convection phenomenon in porous media: effect of Vadasz inertia term” by F. Capone, R. De Luca, M. Vitiello, Ricerche Mat. 68, 581-595 (2019), are recalled in order to better explore the dynamic of thermosolutal convection in a horizontal porous layer with the influence of Vadasz and Soret terms.
\end{abstract}

Keywords Steady convection · Hopf convection · Vadasz $\cdot$ Soret $\cdot$ Stability $\cdot$ Porous media

Mathematics Subject Classification $76 \mathrm{E} 06 \cdot 76 \mathrm{~S} 05 \cdot 35 \mathrm{~B} 35$

\section{Introduction}

In this note we recall the results on the onset of thermosolutal convection in a horizontal porous layer, uniformly heated and salted from below, with Soret and inertia effects, investigated in [1], with particular regard to analyze the kind of instability arising when the rest state is no longer observable. Denoting by $I_{1 n}, I_{2 n}, I_{3 n}$ the principal invariants of the linear operator governing the evolution of the nth component of perturbation,

F. Capone

fcapone@unina.it

R. De Luca

roberta.deluca@unina.it

M. Vitiello

maria.vitiello@poliba.it

1 Department of Mathematics and Applications R. Caccioppoli, University of Naples Federico II, via Cintia, 80126 Naples, Italy

2 Department of Mechanics Mathematics and Management, Politecnico di Bari, via Orabona 4, 70125 Bari, Italy 
the spectral equation is found to be

$$
\lambda^{3}-I_{1 n} \lambda^{2}+I_{2 n} \lambda-I_{3 n}=0
$$

The Routh-Hurwitz conditions, necessary and sufficient to guarantee that all the roots of (1) have negative real part, are

$$
\mathrm{I}_{3 n}<0, \quad \mathrm{I}_{1 n} \mathrm{I}_{2 n}-\mathrm{I}_{3 n}<0
$$

since, for the problem under consideration, $I_{1 n}<0$. These conditions are necessary and sufficient to guarantee that the thermal conduction solution is stable. Convection arises via a steady or oscillatory state if (1) admits the null root or a pure imaginary root: the lowest positive Rayleigh thermal number vanishing $I_{3 n}$ is the Rayleigh thermal number $R_{S}$ for marginal steady state while the lowest positive Rayleigh thermal number such that $I_{1 n} I_{2 n}-I_{3 n}=0$, is the Rayleigh thermal number $R_{O}$ for marginal oscillatory state. The instability threshold is given by $R_{\text {insta }}=\min \left\{R_{S}, R_{O}\right\}$. On comparing these two numbers, in [1], sufficient conditions guaranteeing the onset of steady and Hopf convection have been found. In this short paper we reconsider the problem in [1] and perform the linear instability analysis of the thermal conduction solution of model (1) of [1] by using a different methodology. In this way, we are able to better state the results found in Theorem 1. Precisely, we are able to find conditions guaranteeing that oscillatory convection can not occur because $R_{O}$ does not exist or because $R_{O}$ exists and $R_{O}>R_{S}$.

\section{Linear instability analysis}

The linearized version of (4) in [1] is

$$
\left\{\begin{array}{l}
\frac{1}{\tilde{V}_{a}} \frac{\partial \hat{\mathbf{u}}}{\partial t}+\hat{\mathbf{u}}=-\nabla \hat{\Pi}+R_{T} \hat{\theta} \mathbf{k}-\frac{R_{C}}{L e} \hat{\Gamma} \mathbf{k} \\
\nabla \cdot \hat{\mathbf{u}}=0 \\
\frac{\partial \hat{\theta}}{\partial t}=\hat{w}+\Delta \hat{\theta} \\
\omega \frac{\partial \hat{\Gamma}}{\partial t}=\hat{w}+\frac{1}{L e} \Delta \hat{\Gamma}+S_{r} \Delta \hat{\theta}
\end{array}\right.
$$

The third component of the double curl of (3) $)_{1}$ gives

$$
\frac{1}{\tilde{V}_{a}} \frac{\partial \Delta \hat{w}}{\partial t}+\Delta \hat{w}=R_{T} \Delta_{1} \hat{\theta}-\frac{R_{C}}{L e} \Delta_{1} \hat{\Gamma}
$$


where $\hat{\mathbf{u}}=(\hat{u}, \hat{v}, \hat{w})$ and $\Delta_{1}$ is the two-dimensional Laplacian operator in the horizontal plane.

Looking for normal type solutions, in view of the periodicity in the horizontal directions, we set

$$
\left(\begin{array}{l}
\hat{w} \\
\hat{\theta} \\
\hat{\Gamma}
\end{array}\right)=e^{\sigma t+i a_{x} x+a_{y} y}\left(\begin{array}{c}
\bar{w}(z) \\
\bar{\theta}(z) \\
\bar{\Gamma}(z)
\end{array}\right)
$$

with $\sigma \in \mathbb{C}$. Hence, in view of the boundary conditions

$$
\hat{w}=\hat{\theta}=\hat{\Gamma}=0, \quad \text { on } \quad z=0,1,
$$

one can choose

$$
\left(\begin{array}{l}
\bar{w} \\
\bar{\theta} \\
\bar{\Gamma}
\end{array}\right)=\left(\begin{array}{l}
w_{0} \\
\theta_{0} \\
\Gamma_{0}
\end{array}\right) \sin n \pi z, \quad n \in \mathbb{N}
$$

with $w_{0}, \theta_{0}, \Gamma_{0}$ constants and (4), (3) $)_{3}-(3)_{4}$ become

$$
\left\{\begin{array}{l}
\frac{\xi_{n} \sigma}{\tilde{V}_{a}} w_{0}+\xi_{n} w_{0}=R_{T} a^{2} \theta_{0}-\frac{R_{C}}{L e} a^{2} \Gamma_{0}, \\
\sigma \theta_{0}=w_{0}-\xi_{n} \theta_{0} \\
\omega \sigma \Gamma_{0}=w_{0}-\frac{\xi_{n}}{L e} \Gamma_{0}-S_{r} \xi_{n} \theta_{0}
\end{array}\right.
$$

being $a^{2}=a_{x}^{2}+a_{y}^{2}, \xi_{n}=a^{2}+n^{2} \pi^{2}$. Requiring the vanishing of the determinant of the coefficient matrix in (8), one has

$$
R_{T}=\frac{\xi_{n}+\sigma-S_{r} \xi_{n}}{\xi_{n}+\sigma \omega L e} R_{C}+\frac{\xi_{n}\left(\xi_{n}+\sigma\right)\left(\tilde{V}_{a}+\sigma\right)}{a^{2} \tilde{V}_{a}}
$$

\subsection{Rayleigh number for a steady marginal state}

The Rayleigh number for a steady marginal state, is obtained by (9) on substituting $\sigma=0$ and looking for the minimum with respect to $\left(n, a^{2}\right) \in \mathbb{N} \times \mathbb{R}^{+}$. Then, it is given by

$$
R_{S}=\left(1-S_{r}\right) R_{C}+\min _{\left(n, a^{2}\right) \in \mathbb{N} \times \mathbb{R}^{+}} \frac{\xi_{n}^{2}}{a^{2}},
$$

i.e.

$$
R_{S}=\left(1-S_{r}\right) R_{C}+4 \pi^{2}
$$




\subsection{Rayleigh number for an oscillatory marginal state}

In order to determine $R_{O}$, let us set $\sigma=i \sigma_{1}, \sigma_{1} \in \mathbb{R} \backslash\{0\}$ ( $i$ being the imaginary unit) in (9). Then

$$
R_{T}=\operatorname{Re}\left(R_{T}\right)+i \sigma_{1} \operatorname{Im}\left(R_{T}\right),
$$

with

$$
\operatorname{Re}\left(R_{T}\right)=\frac{\xi_{n}^{2}\left(1-S_{r}\right)+\sigma_{1}^{2} \omega L e}{\xi_{n}^{2}+\sigma_{1}^{2} \omega^{2} L e^{2}} R_{C}+\frac{\xi_{n}\left(\xi_{n} \tilde{V}_{a}-\sigma_{1}^{2}\right)}{a^{2} \tilde{V}_{a}}
$$

and

$$
\operatorname{Im}\left(R_{T}\right)=\frac{\xi_{n}-\omega L e \xi_{n}\left(1-S_{r}\right)}{\xi_{n}^{2}+\sigma_{1}^{2} \omega^{2} L e^{2}} R_{C}+\frac{\xi_{n}\left(\tilde{V}_{a}+\xi_{n}\right)}{a^{2} \tilde{V}_{a}}
$$

Requiring $\operatorname{Im}\left(R_{T}\right)=0$, it follows that $\sigma_{1}^{2}$ has to verify

$$
\omega^{2} L e^{2}\left(\tilde{V}_{a}+\xi_{n}\right) \sigma_{1}^{2}+\left[1-\omega L e\left(1-S_{r}\right)\right] a^{2} \tilde{V}_{a} R_{C}+\xi_{n}^{2}\left(\tilde{V}_{a}+\xi_{n}\right)=0
$$

Remark 1 Equation (15) has no real solution if

$$
S_{r} \geq \frac{\omega L e-1}{\omega L e}
$$

or if

$$
\left\{\begin{array}{l}
S_{r}<\frac{\omega L e-1}{\omega L e} \\
R_{C} \leq \frac{\xi_{n}^{2}\left(\tilde{V}_{a}+\xi_{n}\right)}{a^{2} \tilde{V}_{a}\left[\omega \operatorname{Le}\left(1-S_{r}\right)-1\right]}
\end{array}\right.
$$

Hence, in the cases (16) or (17), $R_{O}$ does not exist and only steady convection can occur.

Remark 2 Simple calculation shows that $(17)_{2}$ is satisfied $\forall\left(n, a^{2}\right) \in \mathbb{N} \times \mathbb{R}^{+}$iff

$$
R_{C} \leq \min _{\left(n, a^{2}\right) \in \mathbb{N} \times \mathbb{R}^{+}} \frac{\xi_{n}^{2}\left(\tilde{V}_{a}+\xi_{n}\right)}{a^{2} \tilde{V}_{a}\left[\omega L e\left(1-S_{r}\right)-1\right]}
$$

i.e. iff

$$
R_{C} \leq \bar{R}_{C}:=\frac{\left(\bar{a}^{2}+\pi^{2}\right)^{2}\left(\tilde{V}_{a}+\bar{a}^{2}+\pi^{2}\right)}{\bar{a}^{2} \tilde{V}_{a}\left[\omega \operatorname{Le}\left(1-S_{r}\right)-1\right]}
$$




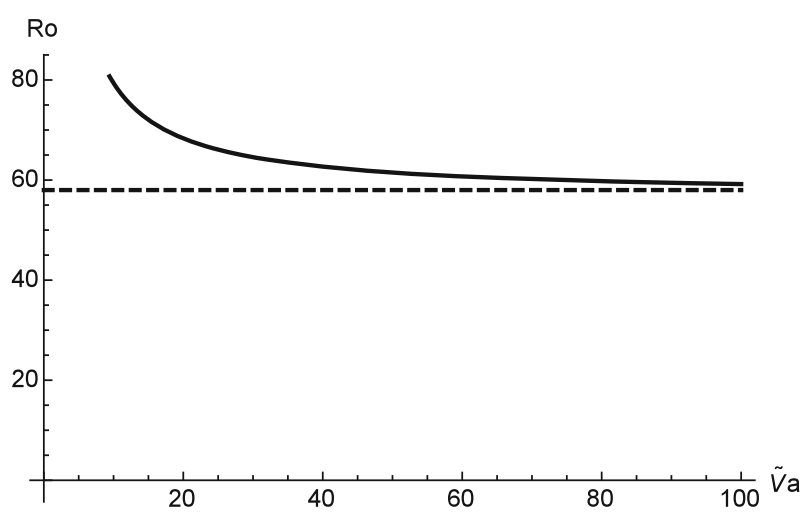

Fig. 1 Asymptotic behaviour of $R_{O}$ for $L e=20 ; \omega=0.2 ; S_{r}=0.5 ; R_{C}=30$ (continuous line); Dotted line denotes $R_{O}$ for $\tilde{V}_{a} \rightarrow \infty$

with $\bar{a}^{2}$ unique positive solution of

$$
2 x^{3}+\left(\tilde{V}_{a}+2 \pi^{2}\right) x^{2}-\pi^{4}\left(\tilde{V}_{a}+\pi^{2}\right)=0 .
$$

In view of (16)-(17) and Remark 1, the condition

$$
S_{r}<\frac{\omega L e-1}{\omega L e}, \quad R_{C}>\bar{R}_{C}
$$

is necessary for the occurrence of Hopf convection. When (21) holds, (15) admits the solution

$$
\sigma_{1}^{2}=\frac{\left[\omega L e\left(1-S_{r}\right)-1\right] a^{2} \tilde{V}_{a} R_{C}-\xi_{n}^{2}\left(\tilde{V}_{a}+\xi_{n}\right)}{\omega^{2} L e^{2}\left(\tilde{V}_{a}+\xi_{n}\right)} .
$$

Substituting (22) into (13) and looking for the minimum, with respect to $\left(n, a^{2}\right) \in$ $\mathbb{N} \times \mathbb{R}^{+}$, one has

$$
R_{O}=\min _{\left(n, a^{2}\right) \in \mathbb{N} \times \mathbb{R}^{+}} g\left(n, a^{2}\right)
$$

with

$$
g\left(n, a^{2}\right)=\frac{\xi_{n}\left(1+S_{r} \omega L e\right)+\tilde{V}_{a} \omega L e}{\omega^{2} L e^{2}\left(\tilde{V}_{a}+\xi_{n}\right)} R_{C}+\frac{\xi_{n}^{2}}{a^{2}} \frac{\omega L e+1}{\omega L e}+\frac{\xi_{n}^{3}}{a^{2}} \frac{\omega L e+1}{\tilde{V}_{a} \omega^{2} L e^{2}} .
$$

The instability threshold is given by

$$
R_{\text {insta }}=\min \left\{R_{S}, R_{O}\right\}
$$

and coincides with that one found in [1]. 
Fig. 2 Asymptotic behaviour of $R_{O}$ versus $\tilde{V}_{a}$ for

$L e=20 ; \omega=0.2 ; R_{C}=30$

and $S_{r}=0.5$ (continuous line);

$S_{r}=0.6$ (dashed line); $S_{r}=0.7$

(dotted line)

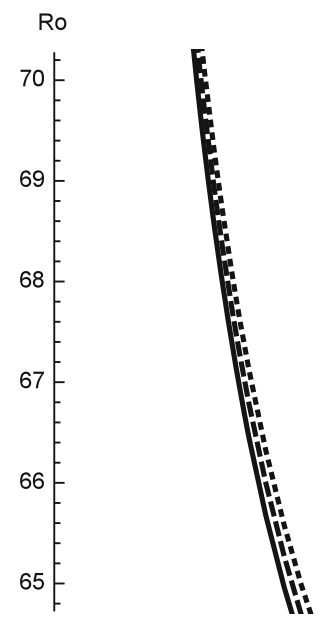

Remark 3 We remark that, since

$$
\min _{a^{2}} \frac{\left(a^{2}+\pi^{2}\right)^{2}\left(\tilde{V}_{a}+a^{2}+\pi^{2}\right)}{a^{2}}=\frac{\left(\bar{a}^{2}+\pi^{2}\right)^{2}\left(\tilde{V}_{a}+\bar{a}^{2}+\pi^{2}\right)}{\bar{a}^{2}},
$$

with $\bar{a}^{2}$ positive solution of (20), then

$$
\bar{R}_{C}<R_{C_{1}},
$$

with $R_{C_{1}}$ (determined in [1]) given by

$$
R_{C_{1}}=\frac{\left(a_{1}^{2}+\pi^{2}\right)^{2}\left(\tilde{V}_{a}+a_{1}^{2}+\pi^{2}\right)}{a_{1}^{2} \tilde{V}_{a}\left[\omega \operatorname{Le}\left(1-S_{r}\right)-1\right]}
$$

In view of Remarks 1, 3 and Theorem 1 in [1], one can conclude that when $R_{T}<R_{C_{1}}$ convection can arise only via a steady state. In particular:

$$
\begin{aligned}
& R_{T}<\bar{R}_{C} \Rightarrow \nexists R_{O}, \\
& \bar{R}_{C}<R_{T}<R_{C_{1}} \Rightarrow \exists R_{O}>R_{S} .
\end{aligned}
$$

\section{Discussion}

In this short paper, we reconsider the onset of thermosolutal convection in a horizontal porous layer, uniformly heated and salted from below, with Soret and Vadasz inertia terms effect, investigated in [1]. Via a different methodology, some analytical results to perform the linear instability analysis of the rest state, found in [1], have been deeply examined. In particular, we have better explored the analytical results found in Theorem 1 of [1]. Precisely, we have been able to specify that, when $R_{T}<R_{C_{1}}$, 
Fig. 3 Asymptotic behaviour of wave number $a_{c}^{2}$ versus $\tilde{V}_{a}$ for $L e=20 ; \omega=0.2 ; R_{C}=30$ and $S_{r}=0.5$ (continuous line); $S_{r}=0.6$ (dashed line); $S_{r}=0.7$ (dotted line)
Table 1 Oscillatory Rayleigh critical numbers in the presence and in the absence of Vadasz inertia term for $R_{C}=50$

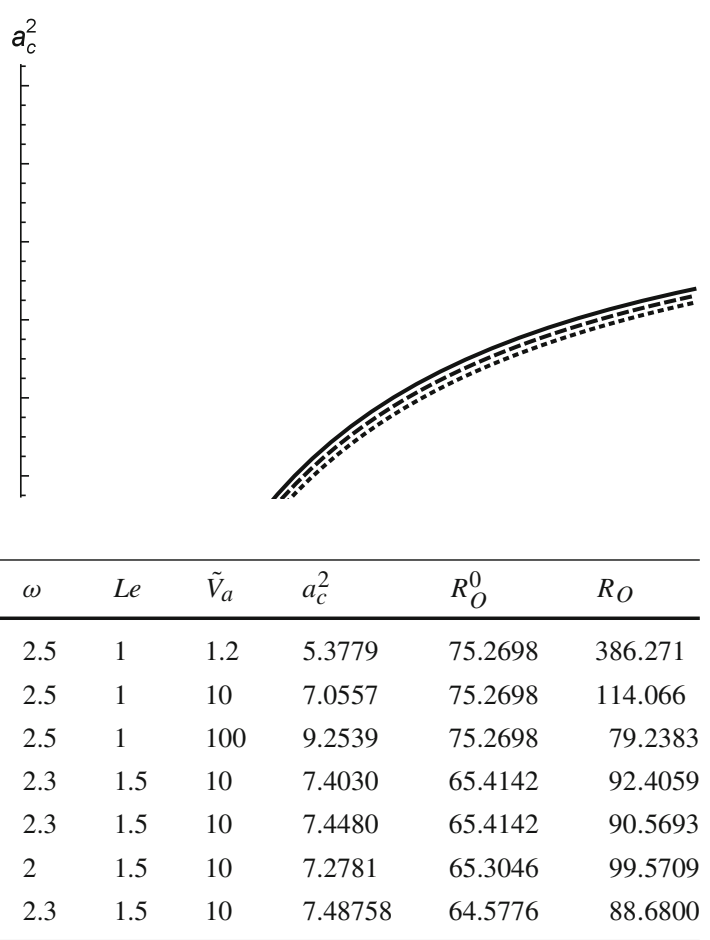

thermosolutal convection can arise only via a steady state since or $R_{O}$ does not exist or $R_{O}$ exists but $R_{O}>R_{S}$. In view of the obtained results, the numerical simulations given in Section 6 of [1] have to be restated. We write in the following, the right version.

$R_{O}$ reverts to the critical Rayleigh number for the onset of Hopf convection in the absence of Vadasz inertia term as illustrated in Fig. 1.

The behaviour of $R_{O}$ versus $\tilde{V}_{a}$ is illustrated in Fig. 2 on fixing $L e=20 ; \omega=$ $0.2, R_{C}=30$ and letting $S_{r}$ vary. The numerical simulations show that $R_{O}$ decreases with respect to $\tilde{V}_{a}$ and increases with respect to $S_{r}$.

Figure 3 shows the wave number for Hopf convection behaviour versus $\tilde{V}_{a}$ on fixing $L e=20 ; \omega=0.2, R_{C}=30$ and letting $S_{r}$ vary. In Table 1 the critical Rayleigh numbers for the onset of oscillatory convection in the absence and in the presence of Vadasz inertia term denoted respectively by $R_{O}^{0}$ and $R_{O}$ and the wave number $a_{c}^{2}$, are collected for $R_{C}=50$ and for different values of $L e, \omega, S_{r}, \tilde{V}_{a} . R_{O}$ decreases with $\tilde{V}_{a}$ (lines 1-3) while increases with $S_{r}$ (lines 4-5). Lines 5-6 show that, as one is expected, $R_{O}$ decreases with $\omega$. In fact, $\omega$ is proportional to the porosity of the medium and, the larger the pores, the more convection is inhibited (i.e. increasing in porosity has a stabilizing effect). Lines 5, 7 concern the behaviour of $R_{O}$ versus $L e$ and, in particular, one can remark that $L e$ has a stabilizing effect on the onset of oscillatory convection.

Acknowledgements This work has been performed under the auspices of the G.N.F.M. of INdAM. The Authors strongly thank Prof. BM. Shankar for helping in improving the quality of the original paper. One 
of the authors (R. De Luca) acknowledges Progetto Giovani GNFM 2020 "Problemi di convezione in nanofluidi e in mezzi porosi bidispersivi”.

Author Contributions All the Authors equally contribute to this work.

Funding Open access funding provided by Università degli Studi di Napoli Federico II within the CRUICARE Agreement.

\section{Declarations}

Conflict of interest The authors declare that they have no conflicts of interest.

Open Access This article is licensed under a Creative Commons Attribution 4.0 International License, which permits use, sharing, adaptation, distribution and reproduction in any medium or format, as long as you give appropriate credit to the original author(s) and the source, provide a link to the Creative Commons licence, and indicate if changes were made. The images or other third party material in this article are included in the article's Creative Commons licence, unless indicated otherwise in a credit line to the material. If material is not included in the article's Creative Commons licence and your intended use is not permitted by statutory regulation or exceeds the permitted use, you will need to obtain permission directly from the copyright holder. To view a copy of this licence, visit http://creativecommons.org/licenses/by/4.0/.

\section{Reference}

1. Capone, F., De Luca, R., Vitiello, M.: Double-diffusive Soret convection phenomenon in porous media: effect of Vadasz inertia term. Ricerche Mat. 68, 581-595 (2019)

Publisher's Note Springer Nature remains neutral with regard to jurisdictional claims in published maps and institutional affiliations. 